\title{
Serum Cholinesterase Is Inversely Associated with Body Weight Change in Men Undergoing Routine Health Screening
}

\author{
Eiji Oda
}

\begin{abstract}
Objective The purpose of this study is to investigate the relationships between serum cholinesterase and body weight change, in addition to incident obesity defined as a body mass index (BMI) of $25 \mathrm{~kg} / \mathrm{m}^{2}$ or greater.

Methods A retrospective 5-year follow-up study was conducted. The crude incidence and hazard ratios (HRs) of obesity adjusted for the BMI and other confounders were calculated for cholinesterase quartiles in 1,412 men and 921 women. Partial correlation coefficients (PCCs) were calculated between cholinesterase and changes in the BMI during the 5-year follow-up period adjusted for age and other confounders and the change in the BMI were compared among cholinesterase quartiles in 1,223 men and 681 women.

Results During the 5-year follow-up period, 149 men (10.6\%) and 65 women (7.1\%) developed obesity. The adjusted HRs of obesity decreased, although the crude incidence of obesity increased along the quartiles of cholinesterase in men. The adjusted HRs of obesity for the first (lowest), second and third quartiles of cholinesterase were $2.02(\mathrm{p}=0.006), 1.45(\mathrm{p}=0.122)$, and $1.28(\mathrm{p}=0.265)$, respectively compared with the highest quartile in men. The PCC between the baseline level of cholinesterase and change in the BMI was $-0.16(\mathrm{p}<0.001)$ in men. The mean changes in BMI for 5 years were $0.31 \mathrm{~kg} / \mathrm{m}^{2}, 0.17 \mathrm{~kg} / \mathrm{m}^{2}, 0.01 \mathrm{~kg} / \mathrm{m}^{2}$ and $-0.04 \mathrm{~kg} / \mathrm{m}^{2}$, respectively in the first, second, third and fourth quartiles of cholinesterase in men $(\mathrm{p}=0.005)$. Neither incident obesity nor weight gain was significantly associated with cholinesterase in women.

Conclusion The serum cholinesterase level was inversely associated with body weight change, as well as incident obesity, after adjusted for the BMI in men.
\end{abstract}

Key words: cholinesterase, body mass index, body weight change, incident obesity

(Intern Med 54: 2427-2432, 2015)

(DOI: 10.2169/internalmedicine.54.4335)

\section{Introduction}

Hippocrates wrote "Corpulence is not only a disease itself, but the harbinger of others", recognizing that obesity is a medical disorder that also leads to many comorbidities (1). The WHO accepts a body mass index (BMI) of $25 \mathrm{~kg} / \mathrm{m}^{2}$ or higher as abnormal; the overweight category is classified as obesity when the BMI is $30 \mathrm{~kg} / \mathrm{m}^{2}$ or more (2). Overweight and obesity represent a rapidly growing threat to the health of populations in an increasing number of countries. Indeed, these states are now so common that they are replacing more traditional problems, such as malnutrition and infectious diseases, as the most significant causes of ill-health. Comorbidities linked to obesity and being overweight include coronary heart disease, hypertension, stroke, certain types of cancer, type 2 diabetes, gallstones, dyslipidemia, osteoarthritis, gout, and pulmonary diseases, including sleep apnea (2). Excess body weight is now the sixth most important risk factor contributing to the overall burden of disease worldwide (3) and abdominal obesity is one of the major components of metabolic syndrome (4). Authorities view it as one of the most serious public health problems of the 21 st century (5). Obesity is most commonly caused by a 
combination of excessive food energy intake, lack of physical activity and genetic susceptibility although a few cases are caused primarily by genetic predisposition, endocrine disorders, medications or psychiatric illness. The author preliminary searched for predictors of obesity among health screening tests. No parameter was found to be associated with incident obesity except for cholinesterase, and the association between cholinesterase and incident obesity was complicated. Some genetic studies have suggested that individuals with high serum cholinesterase levels tend to be thinner while cholinesterase synthesis is reported to increase in individuals who gain weight (6-9).

The aim of the present study is to investigate the association between cholinesterase and incident obesity as well as body weight change in a general health screening population.

\section{Materials and Methods}

Obesity was defined to be a BMI of $25 \mathrm{~kg} / \mathrm{m}^{2}$ or greater according to the Japan Society for the Study of Obesity (10) because the prevalence of obesity as defined by a BMI of $30 \mathrm{~kg} / \mathrm{m}^{2}$ or higher is very low in Japan. The National Nutrition Survey (2000) showed the prevalence of being overweight (BMI: $\left.25-29.9 \mathrm{~kg} / \mathrm{m}^{2}\right)$ and obesity $\left(\geq 30 \mathrm{~kg} / \mathrm{m}^{2}\right)$ was $24.5 \%$ and $2.3 \%$, respectively, in men and $17.8 \%$ and $3.4 \%$, respectively, in women aged 20 years or older (11).

\section{Subjects}

Between April 2008 and March 2009, 2,435 men and 1,437 women visited our Medical Check-up Center for annual general health screenings and provided their written informed consent. The subjects were required to complete a questionnaire including questions about their history of coronary heart disease and stroke, smoking, alcohol consumption, physical activity and use of antihypertensive, antidiabetic and antihyperlipidemic medications.

Study 1 investigated the incidence and hazard ratio (HR) of obesity. Excluding subjects with obesity or a history of coronary heart disease and/or stroke at baseline, the possible candidates for Study 1 included 1,732 men and 1,202 women. Among them, 1,412 men and 921 women revisited our Medical Check-up Center for annual health screenings between April 2009 and March 2014 and were enrolled in Study 1.

Study 2 investigated the relationship between the change in the BMI for 5 years and baseline cholinesterase levels. Excluding subjects with a history of coronary heart disease and/or stroke at baseline, 1,223 men and 681 women who revisited our Medical Check-up Center for annual health screenings between April 2013 and March 2014 were enrolled in Study 2.

This study was approved by the ethics committee of Tachikawa Medical Center and the study procedures were in accordance with the ethical standards set forth in the 1964 Declaration of Helsinki and its later amendments.

\section{Measurements}

After an overnight fast, blood samples were obtained to measure the blood levels of fasting glucose, hemoglobin A1c (HbA1c), triglycerides, high-density lipoprotein (HDL) cholesterol, low-density lipoprotein (LDL) cholesterol, uric acid, high-sensitivity C-reactive protein (hs-CRP), leukocyte count, hemoglobin, aspartate aminotransferase, alanine aminotransferase, gamma glutamyltransferase, creatinine and cholinesterase. The chemical assessments were all performed at BML Nagaoka (Nagaoka, Japan), except for the assessment of hs-CRP, which was performed at BML General Laboratory (Tokyo, Japan) with nephelometry using N-latex CRP-2 (Siemens Healthcare Japan, Tokyo, Japan). The detection limit of hs-CRP was $0.02 \mathrm{mg} / \mathrm{L}$ and a value of hsCRP lower than the detection limit was considered to be $0.01 \mathrm{mg} / \mathrm{L}$. Cholinesterase was measured with the UV method using Serotec ChE CL (Serotec, Sapporo, Japan) which uses p-hydroxybenzoil choline as the substrate. HbA1 c was measured with latex aggregation immunoassay using Determiner HbA1c (Kyowa Medex, Tokyo, Japan) and expressed in NGSP\%. LDL cholesterol was measured using a direct surfactant method with Choletest-LDL (Sekisui Medical, Tokyo, Japan). The estimated glomerular filtration rate (GFR) was calculated as follows: estimated GFR ( $\mathrm{mL} / \mathrm{min} /$ $\left.1.73 \mathrm{~m}^{2}\right)=194 \times$ creatinine $^{-1.094} \times$ age $^{-0.287}$ in men, and $194 \times$ creatinine $^{-1.094} \times$ age $^{-0.287} \times 0.739$ in women according to the Japanese Society of Nephrology (12). The blood pressure was automatically measured with MPV-3301 (NIHON KODEN, Tokyo, Japan). The average SBP and DBP values were calculated from two measurements obtained in the sitting position after five minutes of rest for each measurement. Body weight was measured with the subjects wearing light clothes provided by our Medical Checkup Center, and the weight of the clothes was subtracted from the measured body weight. The BMI was calculated as the weight in kilograms divided by the square of the height in meters.

\section{Statistical analysis}

\section{Study 1}

The baseline data of subjects who developed obesity and those who did not were compared. The means were compared with two-sided Student's $t$-tests and percentages were compared with $\chi$-square tests. The comparisons for triglycerides and hs-CRP were performed after log transformation because the distributions of triglycerides and hs-CRP were highly skewed.

The baseline cholinesterase levels were compared between subjects who developed obesity and those who did not after stratification by the BMI ranges: $<20 \mathrm{~kg} / \mathrm{m}^{2}, 20-20.9 \mathrm{~kg} / \mathrm{m}^{2}$, $21-21.9 \mathrm{~kg} / \mathrm{m}^{2}, 22-22.9 \mathrm{~kg} / \mathrm{m}^{2}, 23-23.9 \mathrm{~kg} / \mathrm{m}^{2}$ and $24-24.9$ $\mathrm{kg} / \mathrm{m}^{2}$.

Using Cox regression models in which years were used as a unit of the survival variable, the first diagnosis with obesity in the annual health screenings was ascertained to be the outcome and subjects without the outcome were cen- 
Table 1. Baseline Data Stratified by the Development of Obesity.

\begin{tabular}{|c|c|c|c|c|c|c|c|c|}
\hline & \multicolumn{4}{|c|}{ men } & \multicolumn{4}{|c|}{ women } \\
\hline & all & nondevelopers & developers & $\mathrm{p}^{\mathrm{a}}$ & all & nondevelopers & developers & $\mathrm{p}^{\mathrm{a}}$ \\
\hline $\mathrm{n}$ & 1,412 & 1,263 & 149 & & 921 & 856 & 65 & \\
\hline age (years) & $51.4(9.3)$ & $51.6(9.3)$ & $49.6(9.0)$ & 0.015 & $51.3(9.4)$ & $51.4(9.3)$ & $50.1(9.6)$ & 0.269 \\
\hline BMI $\left(\mathrm{kg} / \mathrm{m}^{2}\right)$ & $22.0(1.8)$ & $21.7(1.7)$ & $24.1(0.9)$ & $<0.001$ & $20.8(2.1)$ & $20.6(2.0)$ & $23.2(1.8)$ & $<0.001$ \\
\hline systolic BP (mmHg) & $119.6(16.8)$ & $119.4(16.8)$ & $121.2(17.2)$ & 0.213 & $110.4(15.6)$ & $110.3(15.7)$ & $112.9(13.6)$ & 0.191 \\
\hline diastolic BP (mmHg) & $76.2(10.4)$ & $76.1(10.4)$ & $77.4(10.6)$ & 0.143 & $69.0(9.8)$ & $68.9(9.9)$ & $70.0(7.8)$ & 0.392 \\
\hline cholinesterase (U/L) & $326(60)$ & $325(60)$ & $335(59)$ & 0.055 & $312(72)$ & $311(72)$ & $320(65)$ & 0.346 \\
\hline fasting glucose $(\mathrm{mg} / \mathrm{dL})$ & $94.6(13.3)$ & $94.45(13.6)$ & $95.3(10.8)$ & 0.477 & $88.5(9.3)$ & $88.6(9.4)$ & $87.8(6.4)$ & 0.528 \\
\hline hemoglobin A1c (\%) & $5.03(0.47)$ & $5.00(0.48)$ & $5.00(0.34)$ & 0.413 & $5.00(0.33)$ & $5.00(0.33)$ & $4.98(0.33)$ & 0.704 \\
\hline triglycerides $(\mathrm{mg} / \mathrm{dL})$ & $110.3(63.2)$ & $107.7(59.6)$ & $131.8(85.0)$ & $<0.001$ & $80.1(46.1)$ & $79.9(46.7)$ & $83.7(37.4)$ & 0.312 \\
\hline HDL cholesterol (mg/dL) & $60.0(14.8)$ & $60.7(14.9)$ & $54.6(12.9)$ & $<0.001$ & $68.5(14.6)$ & $68.7(14.6)$ & $65.9(14.6)$ & 0.136 \\
\hline LDL cholesterol (mg/dL) & $118.7(27.5)$ & $118.3(27.9)$ & $122.3(23.8)$ & 0.096 & $120.2(29.4)$ & $120.1(29.5)$ & $120.4(29.4)$ & 0.956 \\
\hline uric acid $(\mathrm{mg} / \mathrm{dL})$ & $6.0(1.2)$ & $6.0(1.2)$ & $6.3(1.2)$ & $<0.001$ & $4.4(0.9)$ & $4.4(0.9)$ & $4.4(0.6)$ & 0.654 \\
\hline hsCRP (mg/L) & $0.61(2.04)$ & $0.57(1.64)$ & $0.95(4.05)$ & $<0.001$ & $0.41(0.73)$ & $0.41(0.72)$ & $0.52(0.89)$ & 0.034 \\
\hline leukocyte count $\left(/ \mathrm{L}^{-6}\right)$ & $5,460(1,465)$ & $5,423(1,451)$ & $5,774(1,552)$ & 0.006 & $4,773(1,240)$ & $4,760(1,250)$ & $4,948(1,098)$ & 0.241 \\
\hline hemoglobin $(\mathrm{g} / \mathrm{dL})$ & $14.8(1.0)$ & $14.8(1.0)$ & $15.1(1.2)$ & 0.003 & $12.8(1.1)$ & $12.8(1.2)$ & $12.8(1.0)$ & 0.948 \\
\hline AST (U/L) & $23.1(7.6)$ & $23.1(7.8)$ & $22.6(6.1)$ & 0.418 & $21.2(25.9)$ & $21.4(26.8)$ & $19.6(4.5)$ & 0.592 \\
\hline $\operatorname{ALT}(\mathrm{U} / \mathrm{L})$ & $23.6(12.1)$ & $23.4(12.1)$ & $25.2(11.7)$ & 0.085 & $17.7(15.0)$ & $17.7(15.4)$ & $17.7(7.2)$ & 0.986 \\
\hline GGT (U/L) & $47.1(44.7)$ & $46.5(42.4)$ & $52.1(61.1)$ & 0.145 & $23.2(30.6)$ & $23.3(31.6)$ & $21.8(11.5)$ & 0.712 \\
\hline $\mathrm{eGFR}\left(\mathrm{mL} / \mathrm{min} / 1.73 \mathrm{~m}^{2}\right)$ & $78.4(12.8)$ & $78.6(12.6)$ & $77.3(14.0)$ & 0.232 & $79.8(13.0)$ & $79.8(13.0)$ & $79.5(13.3)$ & 0.857 \\
\hline antihypertensive drugs & 14.3 & 13.8 & 18.8 & 0.098 & 8.6 & 8.5 & 9.2 & 0.845 \\
\hline antidiabetic drugs & 2.3 & 2.5 & 1.3 & 0.573 & 0.5 & 0.4 & 3.1 & 0.045 \\
\hline antihyperlipidemic drugs & 6.6 & 6.1 & 10.7 & 0.031 & 9.8 & 9.8 & 9.2 & 0.879 \\
\hline physical activity $^{\mathrm{b}}$ & 35.8 & 35.7 & 36.2 & 0.898 & 37.9 & 38.8 & 26.2 & 0.043 \\
\hline current smoking & 33.9 & 32.5 & 45.0 & 0.002 & 6.0 & 6.3 & 1.5 & 0.196 \\
\hline daily drinking & 53.8 & 54.2 & 49.7 & 0.290 & 15.1 & 15.2 & 13.8 & 0.771 \\
\hline
\end{tabular}

sored at their last visits; hazard ratios (HRs) of incident obesity were calculated for each $1 \mathrm{U} / \mathrm{L}$ increase in the baseline cholinesterase levels after adjusting for the BMI, age, antihypertensive, antidiabetic, and/or antihyperlipidemic drug use, current smoking, daily alcohol intake and physical activity. Physical activity was defined to be walking for one hour or longer per day or exercising for 30 minutes or longer twice or more per week.

The crude incidence of obesity was calculated for each quartile of cholinesterase. The HRs [95\% confidence intervals (CIs)] of obesity for the lower quartiles of cholinesterase were calculated compared with the highest quartile adjusting for the BMI, age, antihypertensive, antidiabetic, and/or antihyperlipidemic drug use, current smoking, daily alcohol intake and physical activity.

\section{Study 2}

Partial correlation coefficients (PCCs) between the baseline cholinesterase levels as well as the change in cholinesterase levels during the 5-year period, and the baseline BMI, as well as the change in the BMI during the 5year period, were calculated adjusted for age, antihypertensive, antidiabetic, and/or antihyperlipidemic drug use, current smoking, daily alcohol intake and physical activity. The change in the BMI for 5 years was compared among the quartiles of baseline cholinesterase levels using an ANOVA.

All calculations were performed using the Dr-SPSS-2 software program (IBM Japan, Tokyo, Japan). p values of less than 0.05 were considered to be statistically significant.

Results

\section{Study 1}

During the 5-year follow-up period, 149 men (10.6\%) and 65 women $(7.1 \%)$ developed obesity. The baseline data of the obesity developers and non-developers are shown in Table 1 . The BMI, triglycerides, uric acid, hs-CRP, leukocyte count and hemoglobin were significantly higher and age and HDL cholesterol were significantly lower in the obesity developers than non-developers in men while the BMI and hsCRP were significantly higher in the obesity developers than non-developers in women.

The baseline cholinesterase levels of subjects who developed obesity and those who did not is presented in Table 2 stratified by the BMI ranges. The baseline cholinesterase level was significantly lower in subjects who developed obesity than those who did not in the BMI of $24.0-24.9 \mathrm{~kg} / \mathrm{m}^{2}$ group. Otherwise, the baseline cholinesterase levels were not significantly different.

The crude incidence of obesity and the HR of incident obesity for the quartiles of cholinesterase are presented in Table 3. The adjusted HR of obesity significantly decreased although the crude incidence of obesity significantly increased along the quartiles of cholinesterase. The adjusted HR [95\% CI] of obesity for the first (lowest), second and third quartiles of cholinesterase was 2.02 [1.22-3.33] $(\mathrm{p}=$ $0.006), 1.45[0.91-2.32](\mathrm{p}=0.122)$ and 1.28 [0.83-1.99] $(\mathrm{p}=$ 0.265 ), respectively compared with the highest quartile in men. The HRs of obesity for the lower quartiles were not 
Table 2. Baseline Cholinesterase Levels Stratified by Ranges of Body Mass Index (BMI).

\begin{tabular}{|c|c|c|c|c|c|}
\hline & \multicolumn{2}{|c|}{ obesity developers } & \multicolumn{2}{|r|}{ nondevelopers } & \multirow[t]{2}{*}{$\mathrm{p}$} \\
\hline & $\mathrm{n}$ & cholinesterase (U/L) & $\mathrm{n}$ & cholinesterase (U/L) & \\
\hline $\mathrm{BMI}<20 \mathrm{~kg} / \mathrm{m}^{2}$ & 6 & $289.7(30.5)$ & 482 & $298.8(67.5)$ & 0.742 \\
\hline $20 \mathrm{~kg} / \mathrm{m}^{2} \leq \mathrm{BMI}<21 \mathrm{~kg} / \mathrm{m}^{2}$ & 6 & $302.3(58.4)$ & 337 & $312.2(63.2)$ & 0.706 \\
\hline $21 \mathrm{~kg} / \mathrm{m}^{2} \leq \mathrm{BMI}<22 \mathrm{~kg} / \mathrm{m}^{2}$ & 4 & $352.0(54.9)$ & 396 & $318.0(55.3)$ & 0.221 \\
\hline $22 \mathrm{~kg} / \mathrm{m}^{2} \leq \mathrm{BMI}<23 \mathrm{~kg} / \mathrm{m}^{2}$ & 15 & $323.6(89.8)$ & 400 & $332.0(64.6)$ & 0.628 \\
\hline $23 \mathrm{~kg} / \mathrm{m}^{2} \leq \mathrm{BMI}<24 \mathrm{~kg} / \mathrm{m}^{2}$ & 56 & $323.2(49.0)$ & 323 & $334.3(64.7)$ & 0.221 \\
\hline $24 \mathrm{~kg} / \mathrm{m}^{2} \leq \mathrm{BMI}<25 \mathrm{~kg} / \mathrm{m}^{2}$ & 127 & $337.1(62.8)$ & 141 & $352.6(60.3)$ & 0.041 \\
\hline
\end{tabular}

Table 3. Crude Incidence and Hazard Ratio of Obesity by the Quartiles of Cholinesterase.

\begin{tabular}{|c|c|c|c|c|c|}
\hline & Q1 & Q2 & Q3 & Q4 & $\mathrm{p}$ for trend \\
\hline \multicolumn{6}{|c|}{ men } \\
\hline $\mathrm{n}$ & 354 & 354 & 347 & 357 & \\
\hline cholinesterase (U/L) & $146-285$ & $286-323$ & $324-359$ & $360-653$ & \\
\hline crude incidence (\%/year) & 1.7 & 1.9 & 2.2 & 2.6 & 0.029 \\
\hline hazard ratio ${ }^{a}$ ( $p$ value) & $2.02(0.006)$ & $1.45(0.122)$ & $1.28(0.265)$ & 1 & 0.004 \\
\hline $95 \%$ confidence interval & $1.22-3.33$ & $\begin{array}{l}\text { 0.91-2.32 } \\
\text { women }\end{array}$ & 0.83-1.99 & 1 & \\
\hline $\mathrm{n}$ & 230 & 230 & 231 & 230 & \\
\hline cholinesterase (U/L) & $120-262$ & $263-301$ & $302-348$ & $349-733$ & \\
\hline crude incidence $(\% /$ year $)$ & 1.0 & 0.9 & 2.4 & 1.3 & 0.123 \\
\hline hazard ratio ${ }^{a}$ ( $p$ value) & $1.51(0.440)$ & $0.95(0.917)$ & $1.93(0.050)$ & 1 & 0.942 \\
\hline $95 \%$ confidence interval & 0.53-4.28 & 0.39-2.36 & $1.00-3.73$ & 1 & \\
\hline
\end{tabular}

Table 4. Partial Correlation Coefficients $(\mathrm{PCCs})^{\mathrm{a}}$ between Each Parameter.

\begin{tabular}{lccrr}
\hline & \multicolumn{2}{c}{ baseline cholinesterase } & \multicolumn{3}{c}{ changes in cholinesterase } \\
& $\mathrm{PCC}^{\mathrm{a}}$ & $\mathrm{p}$ & $\mathrm{PCC}^{\mathrm{a}}$ & $\mathrm{p}$ \\
\hline & \multicolumn{2}{c}{$\operatorname{men}(\mathrm{n}=1,223)$} & & \\
baseline BMI & 0.33 & $<0.001$ & -0.18 & $<0.001$ \\
changes in BMI & -0.16 & $<0.001$ & 0.51 & $<0.001$ \\
& \multicolumn{2}{c}{ women $(\mathrm{n}=681)$} & & \\
baseline BMI & $0.23 \quad<0.001$ & -0.03 & 0.389 \\
changes in BMI & -0.01 & 0.710 & 0.24 & $<0.001$
\end{tabular}

BMI: body mass index, ${ }^{\text {a }}$ adjusted for age, antihypertensive, antidiabetic, and/or antihyperlipidemic drug use, current smoking, daily alcohol drinking and physical activity

significant compared with highest quartile in women.

\section{Study 2}

The PCCs between the baseline cholinesterase levels, as well as the change in cholinesterase during the 5-year period, and the baseline BMI, as well as the change in the BMI during the 5-year period, are presented in Table 4. The baseline cholinesterase level was significantly positively correlated with the baseline BMI in both men and women while significantly inversely correlated with the change in the BMI in men, but not in women. The change in the cholinesterase level was significantly positively correlated with the change in the BMI in both men and women.

The mean change in the BMI during the 5 year period in each quartile of cholinesterase is shown in Table 5. The mean change in the BMI were $0.31 \mathrm{~kg} / \mathrm{m}^{2}, 0.17 \mathrm{~kg} / \mathrm{m}^{2}, 0.01$ $\mathrm{kg} / \mathrm{m}^{2}$ and $-0.04 \mathrm{~kg} / \mathrm{m}^{2}$, respectively in the first (lowest), sec- ond, third and fourth quartiles of cholinesterase in men ( $\mathrm{p}=$ $0.005)$. There were no significant differences in the mean change in the BMI among the quartiles of cholinesterase in women $(\mathrm{p}=0.304)$.

\section{Discussion}

The present retrospective follow-up study demonstrated that the baseline cholinesterase level was inversely associated with incident obesity after adjusting for the baseline BMI, although the crude incidence of obesity significantly increased along the quartiles of the baseline cholinesterase level and weight change was inversely associated with the baseline cholinesterase level in men undergoing routine health screening. No significant association was observed between the baseline cholinesterase level and incident obesity or body weight change in women. The number of women and incidence of obesity in women may have been too small and too low, respectively to determine significance.

Cross-sectional associations between the serum cholinesterase level and obesity as well as metabolic syndrome (4) are well known (13). Decreased cholinesterase levels have been described in liver cirrhosis to be an index of diminished proteosynthetic function while an increased activity of this enzyme was reported in obesity, diabetes, nephrotic syndrome, hyperthyroidism and in hyperlipidemic subjects without obesity (14). A significant correlation was found between the serum cholesterol or triglycerides and 
Table 5. Body Mass Index (BMI) and Changes in BMI for the Quartiles of Cholinesterase.

\begin{tabular}{|c|c|c|c|c|c|}
\hline & Q1 & Q2 & Q3 & Q4 & $\mathrm{p}^{\mathrm{a}}$ \\
\hline \multicolumn{6}{|c|}{ men } \\
\hline $\mathrm{n}$ & 300 & 314 & 302 & 307 & \\
\hline cholinesterase (U/L) & $146-291$ & $292-331$ & $332-373$ & $374-653$ & \\
\hline BMI $\left(\mathrm{kg} / \mathrm{m}^{2}\right)$ & $22.0(2.3)$ & $22.6(2.4)$ & $23.4(2.6)$ & $24.5(3.0)$ & $<0.001$ \\
\hline changes in BMI $\left(\mathrm{kg} / \mathrm{m}^{2}\right)$ & $0.31(1.37)$ & $\begin{array}{c}0.17(1.11) \\
\text { women }\end{array}$ & $0.01(1.15)$ & $-0.04(1.56)$ & 0.005 \\
\hline $\mathrm{n}$ & 170 & 172 & 169 & 170 & \\
\hline cholinesterase (U/L) & $120-267$ & $268-309$ & $310-354$ & $356-733$ & \\
\hline $\operatorname{BMI}\left(\mathrm{kg} / \mathrm{m}^{2}\right)$ & $20.4(2.5)$ & $21.3(2.5)$ & $22.5(3.3)$ & $23.0(3.8)$ & $<0.001$ \\
\hline changes in BMI $\left(\mathrm{kg} / \mathrm{m}^{2}\right)$ & $0.18(1.28)$ & $0.32(1.49)$ & $0.04(1.50)$ & $0.09(1.53)$ & 0.304 \\
\hline
\end{tabular}

cholinesterase, as an expression of lipid metabolism (14). However, a cross-sectional association cannot clarify a cause-effect relationship.

A previous study investigated the cholinesterase activity during fattening in pigs (the same breed) (14). The results indicate a statistically significant increase in the serum cholinesterase level during fattening suggesting an adaptive increase in the hepatic synthesis of this enzyme in response to the increased lipid metabolism (14). Another study in mice observed an increase in the serum cholinesterase levels in lean mice on a high carbohydrate diet and a marked reduction in the cholinesterase activity in the liver of genetically diabetic mice when starved for 24 hours (15). Another animal feeding experiment observed a significant increase in the serum cholinesterase activity in overweight dogs compared with the group of optimal weight dogs (16). These findings suggest that an increase in the serum cholinesterase level is a result of weight gain or obesity, not a cause.

The present study found an inverse association between cholinesterase and body weight change as well as incident obesity after adjusting for the BMI in men. These results suggest an antiobesogenic property of cholinesterase. Cholinesterase catalyzes the hydrolysis of acetylcholine into choline and acetic acid, a reaction necessary to allow a cholinergic neuron to return to its resting state after activation. Therefore, it is theoretically possible that a low level of cholinesterase may prolong the parasympathetic activity and cause an obesogenic autonomic imbalance. However, this is unlikely because acetylcholine is mainly hydrolyzed by acetylcholinesterase, not by butyrylcholinesterase (BuChE) which comprises most of serum cholinesterase. Polymorphisms of BuChE have been reported to be associated with obesity and hypertriglyceridemia in adults and adolescents. Chautard-Freire-Maia et al. suggested that individuals with innately high BuChE activity tend to be thinner and BuChE synthesis is increased in individuals who gain weight (6-9). Carriers of $1914 \mathrm{G}$ allele in the $B u C h E$ gene showed lower mean BuChE activity when compared to the 1914A carriers, while higher means of BMI and triglycerides were found in the $1914 \mathrm{G}$ carriers when compared to the 1914A homozygotes (17). Therefore, it is possible that the $1914 \mathrm{G}$ allele can be interfering in gluconeogenesis, hyperglycemia, lipolysis and body fat distribution which may lead to an increased predisposition to obesity and to a lower ability to maintain metabolic homeostasis (17). An animal study reported that a BuChE knockout mouse became obese on a high-fat diet (18). Another possible explanation is an association between cholinesterase and the thyroid function. It has been previously reported that the serum cholinesterase activity increased to more than $20 \%$ above the normal level in thyrotoxicosis and decreased by approximately $30 \%$ in myxedema; furthermore, the treatment restored the esterase level to the average mean activity of normal as the patient became euthyroid (19). Therefore, a high level of cholinesterase may be associated with a high level of thyroid hormones which have antiobesogenic property.

The plasma BuChE activity was significantly lower in patients with Alzheimer's disease (AD) than in elderly controls and a reduction in the plasma BuChE activity was directly associated with AD progression (20). Multiple regression analyses confirmed that $\mathrm{AD}$ acts as the main factor in plasma BuChE activity reduction and severe stages are related with an even greater reduction (20). These findings suggest that the reduction of plasma BuChE in $\mathrm{AD}$ patients is most likely associated with a feedback mechanism and provides a future potential of using this enzyme as a possible secondary marker for AD (20). A study in middle-aged or elderly men and women (50 years or older) reported that individuals in the lowest quintile of BuChE activity had significantly higher mortality than those in the highest quintile [HRs (95\% CI): all-cause mortality, 1.62 (1.15-2.30); cardiovascular deaths, 1.79 (1.05-3.05)] after adjusted for other predictors of mortality by Cox regression methods (21). The present results suggest an antiobesogenic property of cholinesterase. Cholinesterase may have beneficial effects on $\mathrm{AD}$, obesity-related diseases and mortality, which implies a possible clinical usefulness of cholinesterase as a health marker.

\section{Limitations}

There are several limitations associated with this study. First, the present study was not a prospective study, but rather a retrospective observational study. Among the 2,934 candidate subjects, 602 individuals $(20.5 \%)$ discontinued the 
study; however, the baseline data did not differ between the candidates and followed subjects. In this study, no dietary information was available, except for the habits of smoking and alcohol intake. Residual cofounders may have thus influenced the present results. No significant association was found in women. The number of women in the present study may have been too small to detect statistically significant results considering the very low prevalence of obesity in Japanese women. Therefore, the conclusions of the present study must be considered with caution. Future prospective studies are therefore required to confirm the present results.

\section{Conclusions}

The present study found that cholinesterase was inversely associated with incident obesity after adjusting for BMI; furthermore, the change in BMI was inversely associated with the cholinesterase levels in men undergoing routine health screening. These results indicate that cholinesterase may have an antiobesogenic property.

\section{The author states that he has no Conflict of Interest (COI).}

\section{Acknowledgement}

The author thanks all subjects who participated in the study, the paramedical staff members at our Medical Checkup Center who assisted with the study, and Honorary Prof. Yoshifusa Aizawa for helpful discussions.

\section{References}

1. Haslam DW, James WP. Obesity. Lancet 366: 1197-1209, 2005.

2. WHO. Obesity: preventing and managing the global epidemic. WHO Tech Report Ser 894: 1-253, 2000.

3. Ezzati M, Lopez AD, Rodgers A, et al; Comparative Risk Assessment Collaborating Group. Selected major risk factors and global and regional burden of disease. Lancet 360: 1347-1360, 2002.

4. Oda E. Metabolic syndrome: its history, mechanisms, and limitations. Acta Diabetol 49: 89-95, 2012.

5. Barness LA, Opitz JM, Gilbert-Barness E. Obesity: genetic, molecular, and environmental aspects. Am J Med Genet A 143: 30163034, 2007.

6. Chautard-Freire-Maia EA, Primo-Parmo SL, Picheth G, Lourenço MA, Vieira MM. The C5 isozyme of serum cholinesterase and adult weight. Hum Hered 41: 330-339, 1991.

7. Alcântara VM, Rodrigues LC, Oliveira LC, Chautard-Freire-Maia EA. Association of the CHE2 locus with body mass index and butyrylcholinesterase activity. Hum Biol 73: 587-595, 2001.

8. Alcântara VM, Oliveira LC, Réa RR, Suplicy HL, Chautard-Freire-Maia EA. Butyrylcholinesterase and obesity in individuals with the CHE2 C5+ and CHE2 C5- phenotypes. Int J Obes Relat Metab Disord 27: 1557-1564, 2003.

9. Dantas VG, Furtado-Alle L, Souza RL, Chautard-Freire-Maia EA. Obesity and variants of the GHRL (ghrelin) and BCHE (butyrylcholinesterase) genes. Genet Mol Biol 34: 205-207, 2011.

10. The Examination Committee of Criteria for 'Obesity Disease' in Japan, Japan Society for the Study of Obesity. New criteria for 'obesity disease' in Japan. Circ J 66: 987-992, 2002.

11. Yoshiike N, Kaneda F, Takimoto H. Epidemiology of obesity and public health strategies for its control in Japan. Asia Pacific J Clin Nutr 11 (Suppl 8): S727-S731, 2002.

12. Matsuo S, Imai E, Horio M, et al. Revised equations for estimated GFR from serum creatinine in Japan. Am J Kidney Dis 53: 982992, 2009.

13. Vallianou NG, Evangelopoulos AA, Bountziouka V, et al. Association of butyrylcholinesterase with cardiometabolic risk factors among apparently healthy adults. J Cardiovasc Med 15: 377-383, 2014.

14. Popescu TA, Fekete T, Popescu E, Böjthy I, Laszlo M. Serum pseudocholinesterase activity during experimental fattening. Med Interne 14: 71-73, 1976.

15. Kutty KM, Huang SN, Kean KT. Pseudocholinesterase in obesity: hypercaloric diet induced changes in experimental obese mice. Experientia 37: 1141-1142, 1981.

16. Tvarijonaviciute A, Tecles F, Ceron JJ. Relationship between serum butyrylcholinesterase and obesity in dogs: a preliminary report. Vet J 186: 197-200, 2010.

17. Lima JK, Leite N, Turek LV, et al. 1914G variant of BCHE gene associated with enzyme activity, obesity and triglyceride levels. Gene 532: 24-26, 2013.

18. Li B, Duysen EG, Lockridge O. The butyrylcholinesterase knockout mouse is obese on a high-fat diet. Chem Biol Interact 175: 88-91, 2008.

19. Thompson JC, Whittaker M. Pseudocholinesterase activity in thyroid disease. J Clin Pathol 18: 811-812, 1965.

20. Bono GF, Simão-Silva DP, Batistela MS, et al. Butyrylcholinesterase: $\mathrm{K}$ variant, plasma activity, molecular forms and rivastigmine treatment in Alzheimer's disease in a Southern Brazilian population. Neurochem Int 81: 57-62, 2015.

21. Calderon-Margalit R, Adler B, Abramson JH, Gofin J, Kark JD. Butyrylcholinesterase activity, cardiovascular risk factors, and mortality in middle-aged and elderly men and women in Jerusalem. Clin Chem 52: 845-852, 2006.

(C) 2015 The Japanese Society of Internal Medicine http://www.naika.or.jp/imonline/index.html 